Décadrages Décadrages

cınéma, à travers champs Cinéma, à travers champs

3 | 2004

Hitchcock côté cour

\title{
Hitchcock et l'art contemporain : Remake. A propos d'une bande vidéo de Pierre Huyghe
}

\section{François Bovier}

\section{OpenEdition}

\section{Journals}

Édition électronique

URL : http://journals.openedition.org/decadrages/553

DOI : $10.4000 /$ decadrages.553

ISSN : 2297-5977

Éditeur

Association Décadrages

Édition imprimée

Date de publication : 10 avril 2004

Pagination : 18-34

ISBN : 978-2-9700582-0-5

ISSN : 2235-7823

Référence électronique

François Bovier, « Hitchcock et l'art contemporain : Remake. A propos d'une bande vidéo de Pierre Huyghe », Décadrages [En ligne], 3 | 2004, mis en ligne le 26 novembre 2012, consulté le 19 avril 2019. URL : http://journals.openedition.org/decadrages/553; DOI : 10.4000/decadrages.553

(B) Décadrages 
1 Pierre Huyghe, à vrai dire, tient plutôt à marquer une distance vis-à-vis des films et n'adhère en aucune façon à la cinéphilie. Ainsi affirme-t-il sans ambages lors d'une conversation avec Vincent Dieutre que le cinéma ne constitue pas l'objet de son propos. "Je peux revenir à toute cette problématique de l'art qui aujourd'hui tournerait autour du cinéma. Je ne suis pas un critique de cinéma, je ne fais pas une réflexion sur le cinéma. Je ne suis pas nostalgique du cinéma et je ne fais pas uniquement référence au cinéma; c'est pour moi un outil qui permet de développer quelque chose et ça s'arrête là. Maintenant, avec cette nécessité qu'ont eue les artistes de passer par une expérience temporelle, disons, en tout cas liée à une histoire, à une narration, l'approche du cinéma s'est forcément un peu répandue [...]." Cf. "Dialogue entre Pierre Huyghe et Vincent Dieutre (2 $2^{\mathrm{ème}}$ partie)", in La lettre du cinéma, $n^{\circ} 16$, hiver 2001, p. 32.
Pierre Huyghe, dans Remake (France, 1994-1995, 100'), remet en scène et en cadre Rear Window (Fenêtre sur cour, Etats-Unis, 1954, 112') en faisant appel à des interprètes amateurs qui s'expriment en français et en transposant l'action autour de la cour intérieure d'un immeuble en chantier. En retournant un scénario du "maître du suspense» pour mieux le détourner, Pierre Huyghe relance la question des rapports que l'art contemporain entretient avec le cinéma. Ajoutons, pour être plus précis, qu'il interroge l'engouement d'un certain nombre d'actants de ce champ envers le cinéma dit d'auteur, que la figure de Hitchcock incarne emblématiquement. Et il le fait en prenant pour cas d'espèce, au sein d'un vaste et hétérogène corpus, un film qui met en abîme et thématise l'activité du spectateur de cinéma. Car Rear Window, pour rapporter un lieu commun, est axé sur le voyeurisme et, chose tout aussi rabâchée, sur son envers ou son revers, c'est-à-dire sur la frustration d'un regard dont l'omnipotence s'avère dérisoire. Aussi la focalisation du film estelle relayée et redoublée par des outils d'observation, en premier lieu les jumelles et l'appareil photographique avec son objectif grossissant, qui finissent par se retourner en instruments d'aveuglement (le flash affole le regard du criminel démasqué - qui dans le retournage ne porte plus de lunettes, autre prothèse de la vision).

Pierre Huyghe, en intervenant sur le film de Hitchcock, cherche à déceler les points de convergence et de divergence entre le cinéma et l'art contemporain. Autrement dit, sa démarche tend à inverser le point de vue le plus courant: au lieu de remodeler le cinéma au gré de la logique et des stratégies de l'art contemporain, il guette les frictions et les écarts entre la pratique filmique et le champ des arts visuels ${ }^{1}$. En fin de compte, c'est à une opération de soustraction qu'il se prête: l'ersatz qu'il produit appauvrit l'original. Le plan inaugural de Remake désigne ce qui est visé dans ce déplacement: Pierre Huyghe, au lieu de restituer le premier panoramique qui parcourt la cour, cadre en gros 
plan un thermomètre atmosphérique; en soulignant cet outil de mesure (indiquant $13^{\circ} \mathrm{C}$ : la graduation a été convertie selon l'échelle française), il signifie que Remake s'inscrit dans un art de la surface et de la façade, un cinéma désaffecté à l'image d’un réel désenchanté.

\section{De l'art contemporain au cinéma}

L'actualité de son propos n'en est devenue que plus pressante avec le temps. En témoigne, pour s'attacher au signe le plus manifeste, l'exposition "Hitchcock et l'art», récemment organisée à Beaubourg par Dominique Païni et Guy Cogeval, qui privilégie un fétichisme de l'objet, une mise en valeur et en vitrine des accessoires. Les commissaires d'exposition, provenant à la fois du champ de l'art et du cinéma, semblent répondre à une question posée jadis: "Comment peut-on être hitchcocko-hawksien?»2 En décelant les signes de l'art dans les indices semés épars au gré des plans...

De toute évidence, les commissaires opèrent un glissement entre une thématique liée à un auteur et une tradition iconographique dans laquelle s'inscrit un artiste. A mon sens, s'appliquer à démontrer que Hitchcock mobilise tout un pan de l'histoire de la représentation (peintures et illustrations au premier chef) ${ }^{3}$ revient à céder au démon de l'analogie. Toutefois, on ne peut que constater un mouvement d'interaction et de fécondation entre le cinéma et l'exposition depuis une vingtaine d'années (ce qui a conduit certains critiques à parler de "cinéma d'exposition»). Pour s'en tenir à Hitchcock et à deux possibilités extrêmes, rappelons que Douglas Gordon est intervenu sur la scène du meurtre de Psycho pour la distendre sur une durée de vingt-quatre heures (24 Hour Psycho, 1993), tandis que Matthias Müller et Christoph Girardet ont condensé et redistribué l'ensemble du corpus hitchcockien par entrées thématiques, par nomenclature d'objets, par relevé de lieux et par mise en série de gestes (Phoenix Tapes, 1999, 45'). Ces bandes vidéo reposent sur des mécanismes opposés, mais provoquent toutes deux une tension entre le temps de l'histoire et le temps de la projection : la technique de l'extrême ralenti et la pratique du zapping renouvellent la relation du spectateur au film, en dilatant ou en contractant la durée du visionnement. Je relierais volontiers ce figement du rythme de défilement du film et cette précipitation de l'enchaînement des séquences à l'une des principales motivations de la promotion du cinéma au rang de référent privilégié de l'art contemporain. Car l'enjeu de certaines démarches artistiques (plus particulièrement, l'appropriation et le détournement) porte précisément sur le vecteur temporel du film (une durée linéaire imposée au spectateur) et sur sa propension à relater un récit (dont les codes ont rapidement été établis) 4.
2 La formule renvoie à un article d'André Bazin qui interrogeait la position des "jeunes Turcs" des Cahiers du Cinéma. Cf. André Bazin, "Comment peut-on être Hitchcocko-Hawksien?", in Cahiers du Cinéma, $\mathrm{n}^{\circ}$ 44, février 1955, p. $17-18$.

3 Sur ce point, voir Hitchcock et l'art. Coïncidences fatales, Dominique Païni et Guy Cogeval (éd.), Centre Pompidou/Mazzotta, Paris, 2001. Des toiles de Dante Gabriele Rossetti ou Julia Margaret Cameron, par exemple, côtoient celles de Georges Rouault ou Edward Munch en tant qu'intertextes des films de Hitchcock.

4 Au sujet de la linéarisation et de la narrativisation du cinéma, on consultera l'ouvrage de Noël Burch sur la constitution progressive d'un mode de représentation institutionnel. Cf. Noël Burch, La lucarne de l'infini. Naissance du langage cinématographique, Nathan, Paris, 1991 [première édition anglaise: 1990]. 
5 Craig Owens, "The Allegorical Impulse: Toward a Theory of Postmodernism", in October, $\mathrm{n}^{\circ} 12$ et 13 , printemps et été 1980 , p. $67-86$ et p. 58-80.
L'autre grande raison de l'attrait du cinéma tient, à mon sens, à son mode de représentation qui peut être décrit comme un collage d'éléments disjoints, comme un aboutage de fragments hétérogènes. Sans même faire mention du mode de production collectif des films qui met en crise la notion bourgeoise de l'individualité de l'artiste, le cinéma a été convoqué à titre de comparant pour définir la nature de l'art contemporain. A cet égard, la prise de position de Craig Owens 5 qui fait figure de pionnier dans ce champ me paraît exemplaire. Ce dernier définit l'art contemporain comme une réactivation d'un "élan allégorique» (en référence à la thèse d'habilitation de Walter Benjamin sur l'origine du drame baroque allemand) qui mobilise l'ordre du fragmentaire et la structure du palimpseste pour provoquer un effet d'indécidabilité du sens. Il identifie alors le cinéma à une machinerie qui automatise ces procédés: la fragmentation et l'accumulation des plans, la multiplication des indices signifiants à l'image et leur résistance à l'imposition d'un sens univoque décuplent les effets visés par les praticiens de l'art contemporain. Ainsi, les débats qui se sont engagés dans les années 1980 autour de la logique interne à l'œuvre d'art à l'ère du "postmodernisme» ont eu pour effet de marquer par la bande la porosité des frontières séparant l'art vidéo et l'installation du cinéma expérimental et élargi (dans la mesure où la fragmentation et la reconstitution de l'espace-temps filmique constituent l'objet d'investigation de ces pratiques). Mais revenons à Pierre Huyghe - qui se positionne d'ailleurs plutôt à l'encontre du «postmodernisme» et de son regard nostalgique.

Remake déjoue le forçage du dispositif cinématographique qui peut caractériser l'art de l'appropriation et les limites d'un récit filmique qui répond à des codes préétablis. Le retournage, que l'on peut pragmatiquement rattacher à la pratique des versions multiples (Huyghe tourne bien après-coup une version française, étrangement française, d'un film issu des studios hollywoodiens) ou réinscrire génériquement dans la logique du remake (Huyghe propose une variation sur le film de Hitchcock qu'il réactualise), n'existe qu'en fonction d'un texte qu'il supplée. En spectacularisant cette pratique, Pierre Huyghe met à mal le mythe moderniste de «l'originalité de l'artiste» (et du cinéma d'auteur): il substitue à «l'authenticité» et à «l'originalité» de l'ouvre d'art (à l'exhibition des marques énonciatives d'un cinéma se voulant distancié) le règne du semblable et du multiple.

- C'est ainsi que Rosalind Krauss décrivait l'économie interne de l'art contemporain (ou "d'après le modernisme»). C'est ainsi que Walter Benjamin appréhendait et promulguait les moyens de reproduction mécanique. Qu'on excuse ces truismes. Mais c'est bien à partir de cette 
communauté de lieu que Pierre Huyghe intervient sur Rear Window pour introduire l'écart du jeu entre stratégies de l'art contemporain et pratiques filmiques.

Ajoutons que Remake a justement pour objet de creuser le fossé entre deux modalités de représentation: Pierre Huyghe parasite le format du film hollywoodien par le support de la vidéo amateur ${ }^{6}$. Par ce geste de restitution d'un déjà-vu par-delà un long laps de temps, Pierre Huyghe éveille chez le spectateur une prise de conscience des traits constitutifs de la représentation filmique et des effets qu'elle peut induire (de l'identification à l'aliénation). Je me situe donc, on l'aura compris, aux antipodes du point de vue polémique de Jean-Paul Fargier qui aujourd'hui dénonce les "huygheries», les «gorgonades» et autres gestes d'"Iconoclastes» comme un vain égarement dans le champ du cinéma (sacralisé), conduisant en fin de compte à la dissolution de ce dernier, tout en portant atteinte à la «Fiction», voire au «Réel»7!

\section{Du cinéma aux versions multiples}

Dans le champ interne à la littérature, Jorge Luis Borges avait en son temps pointé les paradoxes d'une reprise littérale: avec «Pierre Ménard, auteur du Quichotte» (1939)8, il démontrait que la réception et l'interprétation d'un texte étaient contingentes, c'est-à-dire dépendantes d'un contexte historique et d'un horizon d'attente générique qui varient avec le temps. Dans une allégorie, donnée comme une réponse à des articles mal informés au sujet de l'œuvre du défunt Pierre Ménard, Borges attribue à cet écrivain imaginaire un ultime projet qui consistait à réécrire, "mot à mot et ligne à ligne», des parties du Don Quichotte de Miguel de Cervantès. Reproduire littéralement en France, au $\mathrm{XX}^{\mathrm{e}}$ siècle, des extraits d'un texte écrit en Espagne, au XVII ${ }^{e}$ siècle, aboutit, aux yeux de Borges, à la constitution d'une œuvre autonome. Plus même, le texte de Ménard serait supérieur à celui de Cervantès, puisque les événements
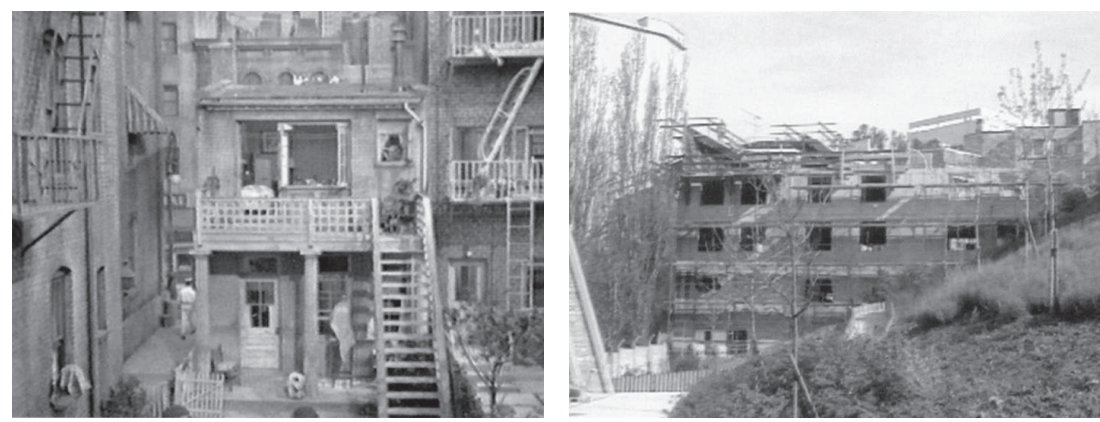

6 Notons que Muriel Caron attribue un double enjeu à la pratique du remake huyghien: "La répétition - au sens de la réactivation - d'une image (d'un objet, d'un son, d'un film, d'un geste, d'un comportement, ...) répond en effet à un double enjeu. D'une part, arracher l'image aux clichés, à la surabondance visuelle, en la caractérisant. D'autre part, retarder l'effet de laminage du temps qui n'aura de cesse d'effacer le contenu de l'image pour ne l'autoriser à témoigner qu'à travers sa forme et son style." Cf. Muriel Caron, "Les Remakes de Pierre Huyghe", in Parachute, $\mathrm{n}^{\circ}$ 93, janvier 1999, p. 4.

7 Cf. Jean-Paul Fargier, "Le bonjour d'Alfred", in Vertigo, $n^{\circ} 25$, printemps 2004, p. 38-45. Fargier s'en prend à l'idée qu'un film déjà réalisé puisse faire l'objet d'un ready-made arrangé (déplacé dans l'espace muséal). II est ainsi amené à dénoncer le "protocole huyghien du retournage à l'identique": "Reconstruire le cinéma dans l'art vidéo n'a aucun sens, sinon celui de dissoudre l'art vidéo: c'est la bévue que commet Pierre Huyghe en réalisant ses remakes. Or refaire, même autrement, n'est pas reconstruire. [...] Les remakes de Huyghe sont des pseudo remakes. En réalité, ce sont des retournages bornés. Au plan près. Tétanisés de mimétisme." (Id., p. 40) Je proposerais le schéma (fourre-tout) suivant pour résumer les termes (bancals) du débat: cinéma d'auteur (Fiction, Réel) / authenticité, originalité, unicité / modernisme vs remake (signifiants désancrés) / secondarité, similitude, reproductibilité / post-modernisme.

8 Jorge Luis Borges, "Pierre Ménard, auteur du Quichotte", Fictions (1960), Gallimard, Paris, 1965 (traduit par P. Vendevoye, Ibarra et Roger Caillois). 
9 La figure du palimpseste est au centre de la réflexion de Craig Owens (op. cit.) sur l'art contemporain. Rappelons sa définition: sur un parchemin, un texte second efface l'original; par extension, une nouvelle inscription supplée la première, comme le Nouveau Testament a pu recouvrir l'Ancien Testament. Craig Owens (qui cite "Pierre Ménard, auteur du Quichotte" dans la première partie de son essai) identifie les prémices du "postmodernisme" et de sa logique allégorique dans les peintures de surface de Robert Rauschenberg (en position de "plateau" horizontal, disait Leo Steinberg) où se déversent pêle-mêle images découpées, objets de rebut et pigments de couleur (il s'agit de la série des Combine-Paitings produites dans les années 1950).

10 Notons que la plupart des pièces de Pierre Huyghe supportent le passage par différents formats ou supports: la projection cinématographique, la diffusion vidéo ou encore l'installation.

11 Borges, dans sa parabole, parle d'extraits de textes reproduits. Les praticiens du found footage sont coutumiers de la chose: des cinéastes aussi différents que Bruce Conner, Morgan Fisher ou Martin Arnold ont réalisé des films sans tourner un seul plan. Mais il semblerait qu'une reprise intégrale d'un film implique un retravail (peut-être pouvons-nous définir en ces termes une tendance du "cinéma structurel»). Ainsi, Ernie Gehr avec Eureka (1974) reproduit au ralenti un film du début du siècle sur San Francisco, tourné depuis un funiculaire. Ou encore Ken Jacobs avec Keaton's Cops (1994) projette bien le film cité dans le titre, mais non sans avoir masqué au préalable les trois-quarts supérieurs de l'image.

12 En deçà de la pratique des versions multiples, la liquidation de l'original par les moyens de reproduction mécanique peut être réfutée pour des raisons contingentes (censure, adaptation selon les pays, initiatives de l'exploitant, usure de la copie, etc.): avec le temps, il n'y a pas deux copies de films qui demeurent identiques, ou peu s'en faut. rapportés sont distanciés et opacifiés: un style archaïque, et dès lors marqué, se substitue à l'espagnol courant; une fiction historique qui se passe dans l'Espagne de Lope de Vega se substitue à l'opposition entre idéaux chevaleresques et réalité provinciale d'une époque. Le paradoxe que nous soumet Borges demeure étroitement lié à l'espace littéraire : cette fiction ne peut revêtir un air de vraisemblance qu'en mobilisant la structure du palimpseste ${ }^{9}$. Pour Borges, la reduplication des mêmes signes efface leur tracé originaire et leur attribue un sens renouvelé. Rapportons sa conclusion:

"Ménard (peut-être sans le vouloir) a enrichi l'art figé et rudimentaire de la lecture par une technique nouvelle: la technique de l'anachronisme délibéré et des attributions erronées. [...] Cette technique peuple d'aventure les livres les plus paisibles. Attribuer l'Imitation de Jésus-Christ à Louis-Ferdinand Céline ou à James Joyce, n'est-ce pas renouveler suffisamment les minces conseils spirituels de cet ouvrage?»

Peut-on symétriquement avancer que Pierre Huyghe, en s'attribuant Rear Window, l'enrichit infiniment? Il semblerait que non: Remake n'est pas assimilable à une reprise littérale ou à une appropriation, à un hommage ou à une métacritique. A filer la métaphore, on pourrait tout au plus soutenir qu'il s'agit, pour Pierre Huyghe, d'exproprier une œuvre originale et d'imposer à sa place un texte apocryphe. En instituant une procédure de comparaison infinie (Pierre Huyghe a parfois projeté simultanément l'original et la copie sur deux écrans superposés verticalement), la répétition introduit un écart différentiel et imprime à l'œuvre une légère torsion $\mathbf{1 0}$. Mais à mobiliser des critères internes au champ cinématographique, les éléments de cette équation d'identité doivent être réajustés. D'une part, le remake constitue une pratique courante qui ne s'apparente pas forcément au pastiche ou à la parodie (et je ne dis pas par là que Borges visait ces seuls phénomènes). De plus, l'hypothèse d'une reprise littérale d'une ouvre constitue un fait avéré dans le cas du cinéma (encore que l'auteur, il faut le concéder, intervient le plus souvent d'une façon ou d'une autre sur l'original) 11. D'autre part, le remake, par définition, et déjà les versions multiples, par voie de fait, introduisent plusieurs variabilités entre textes primaire et secondaire $\mathbf{1 2}$. Pour s'en tenir aux traits les plus saillants, le retournage introduit une double différence: les acteurs, dans les versions multiples par exemple, ne sont plus les mêmes, et si d'aventure ils restaient identiques, ils n'en demeureraient pas moins enclins à des changements gestuels, mimiques et d'articulation; le monde profilmique subit un certain nombre de transformations et de déplacements, en particulier au niveau de ses 

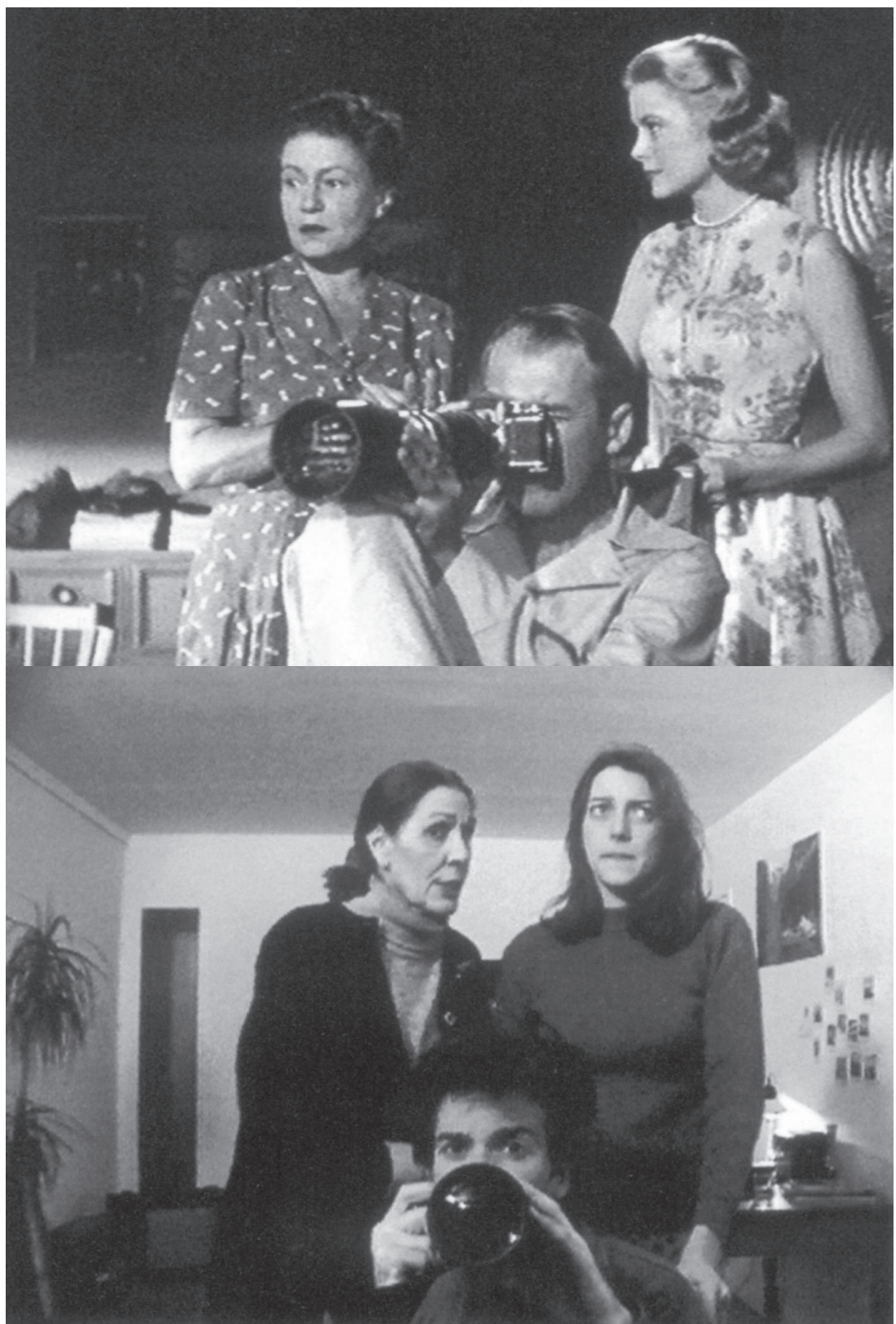
13 Pascale Cassagnau a bien analysé la fonction de l'interprète chez Pierre Huyghe, encore que sur un point je ne saurais la suivre (la référence à Bresson que Huyghe lui-même mobilise volontiers: pourtant, les modèles bressoniens répètent leur rôle jusqu'à l'épuisement et en vue d'obtenir des effets fort calculés): "L'interprète, que Robert Bresson nomme un modèle dans ses Notes sur le cinématographe, a pour caractéristique ontologique de ne pas apparaître en personne, mais d'emprunter une parole ou une identité déléguée pour les restituer en les augmentant, c'est-à-dire en en développant, par "étirements" répétés et progressifs, les potentialités. La partition dont s'empare un interprète pour la réactiver est le support d'une répétition qui finit par produire du singulier; tel est le paradoxe mis en évidence par Nietzsche: la répétition, qu'il désigne comme une rumination, est une opération qui permet d'échapper à la tyrannie du temps et au ressentiment qui l'accompagne." Pascale Cassagnau, "Pierre Huyghe, le temps désaffecté / projet", in Omnibus, $n^{\circ} 19$, janvier 1997, p. 10.

14 Selon Philippe Parreno, Remake a été tourné en deux week-ends. Cf. Pierre Huyghe / The Trial, Lili Fleury, Laurent Godin et Pierre Huyghe (éd.), Kunstverein München, Kunsthalle Zurich, Secession, Le Consortium, 2000, p. 12. Dans une interview, Pierre Huyghe déclarait avoir tourné le film en dix jours. Cf. Dominique Gonzales-Foerster, "Pierre Huyghe, l'état de chantier permanent", in Purple Prose, n ${ }^{\circ}$, été 1995, p. 118. composantes spatio-temporelles. Pierre Huyge, en démultipliant ces facteurs de variabilité, marque l'incommensurabilité entre un film trop souvent vu pour échapper aux automatismes de la reconnaissance et sa propre version arrangée (qui répond au modèle bakhtinien du «travestissement carnavalesque»).

\section{De l'appropriation à la déterritorialisation}

Dégageons enfin les écarts les plus patents qui caractérisent Remake.

$1^{\circ}$ Les acteurs, en imitant tant bien que mal leurs modèles, exhibent leur incapacité à reproduire un jeu qui répond à des codifications de genre. Leurs propos résonnent étrangement et leurs gestes sont dédramatisés 13. De plus, en "doublant» en prise synchrone les voix qui sont par la suite mises à distance à travers un effet de play-back, ils attirent notre attention sur la pratique de la télédiffusion en bi-canal. L'ensemble de ces procédés sera d'ailleurs ausculté par Pierre Huyghe: Casting (1995) se concentre sur les procédures de sélection des acteurs, Dubbing $\left(1996,120^{\prime}\right)$ isole la phase du doublage en reprenant les dialogues d'un film d'horreur (Poltergeist, Tobe Hooper, 1982) et Versions multiples (1997) explore les différences de mise en scène, de cadrage et d'interprétation entre les versions anglaise, allemande et française d'Atlantis (E. A. Dupont, 1929, 90'/100'/85').

$2^{\circ}$ Les lieux sont radicalement altérés: si les décors de Hitchcock, malgré leur relative discrétion à l'écran, ont nécessité des coûts exceptionnels de fabrication, Pierre Huyghe opte pour un décor préexistant au film et en état de chantier (cette dégradation ponctuelle gagne également la bande-son par intermittence: des bruits de marteau-piqueur, de perceuse et de coups de marteau couvrent souvent la voix des interprètes). Aussi l'espace n'est-il pas construit en fonction des prises de vues et des mouvements de caméra, ce qui implique un repositionnement des acteurs et des objets au sein de l'espace profilmique.

$3^{\circ}$ Le support vidéo, qui favorise la réduction de l'équipe technique, rapproche le film du spectateur, en attribuant à l'image les traits caractéristiques du home movie. Car c'est peut-être d'un film de famille qu'il s'agit: tourné en un laps de temps très bref $\mathbf{1 4}$, Remake ausculte les attitudes, les gestes et la diction improvisés d'acteurs qui ne se départissent à aucun moment de leurs idiosyncrasies et de leur spontanéité.

$4^{\circ}$ Le retournage substitue aux référents mondains une représentation seconde. Les interprètes assument le rôle de doublures. Pierre Huyghe s'exprime sans équivoque à ce sujet:

"Je pense [...] à la figure du somnambule, principalement dans le remake. Quelqu'un répète quelque chose sans en avoir conscience. 
N'étant pas affecté par ce qu'il est ou fait, n'essayant pas de rentrer dans la peau d'un personnage, il reste "dans» la surface. Les acteurs - non-professionnels - de mes films sont comme des doublureslumière, ces gens qui se trouvent sur le plateau avant la répétition. [...] L'actrice qui joue le rôle de Grace Kelly dans le remake de Fenêtre sur cour ne va pas s'intéresser à la personne que Grace Kelly interprète mais à la façon dont Grace Kelly interprète ce personnage. [...] Les acteurs ont connaissance du texte quelques heures avant le tournage, ils l'interprètent comme ils peuvent. Ce sont précisément leurs difficultés, hésitations, silences, etc. que j'enregistre en temps réel.»15

Ou encore: "J'ai demandé à mes acteurs de répéter, d'être des doubles, de reproduire»16. Le spectateur, dès lors, n'assiste pas à une narration mais à une (re)mise en scène: palimpseste neutralisé, Remake constitue un acte herméneutique paradoxal, qui n'ajoute ni ne retranche quoi que ce soit (ou si peu) à l'original, mais qui en barre l'accès pour lui opposer un simulacre.

L'ensemble de ces points amenait Jean-Christophe Royoux à comparer Remake à un "karaoké audiovisuel» $\mathbf{1 7}$ où le spectateur est amené à prendre imaginairement la place de l'acteur.

\section{Re-jouer, re-tourner et re-voir: démonter}

Le caractère laborieux de l'interprétation exhibe les mécanismes du remake: chaque action paraît artificielle et ouverte à d'autres modes d'interprétation; les événements, acquérant une relative autonomie, s'égrènent en une succession de moments qui ne parviennent pas à se fondre en un continuum filmique. Bref, ce double mouvement de désinvestissement psychologique du rôle et de dévitalisation de l'original empêche le spectateur de se concentrer sur l'histoire racontée. Ainsi, selon Jean-Christophe Royoux, Pierre Huyghe génère un effet
15 Propos rapportés par Françoise Chalouin, "Pierre Huyghe, Des scénarios pour les temps libres ", in Documents sur l'art, n 9, été 1996, p. 24.

16 Propos rapportés par Jean-Christophe Royoux, "Remaking Cinema", Cinéma Cinéma. Contemporary Art and the Cinematic Experience, Jaap Guldemond (éd.), Stedeljik vam Abbemuseum Eidhoven / NAi Publishers, Rotterdam, 1999, p. 22.

17 lbid.
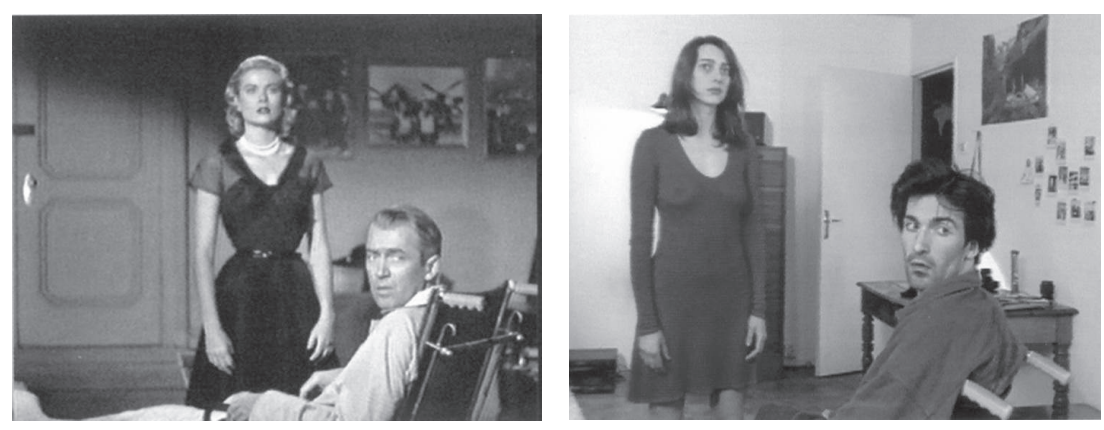
18 Paul Willemen, dans le contexte des prises de position féministes qui dénoncent le voyeurisme et le fétichisme attachés à la vision de films, détermine à partir des courts métrages de Stephen Dwoskin un quatrième regard qui plongerait le spectateur dans l'embarras et qui déréglerait cette économie du désir scopique. Outre le regard de la caméra sur le profilmique, les regards intradiégétiques échangés entre les personnages et le regard du spectateur sur le film (canalisé par les deux précédents, suivant les mécanismes de l'identification primaire et secondaire), il faut parfois encore compter sur le regard du modèle (le plus souvent une actrice qui dévisage la caméra tenue par Dwoskin) qui provoque un sentiment de déplaisir chez le spectateur. Cf. Paul Willemen, "The Fourth Look", Looks and Frictions, Essays in Cultural Studies and Film Studies, British Film Institute / Indiana University Press, Londres / Indiana, 1994 (première publication dans Screen: 1976).

19 A cet égard, on consultera l'essai de JeanCharles Masséra sur The Third Memory qui emprunte lui-même la forme d'un remake de La société du spectacle (Guy Debord). Cf. Jean-Charles Masséra, "La leçon de Stains", Pierre Huyghe. The Third Memory, Centre Pompidou / The Renaissance Society, 2000. La filiation que je propose entre remake et reconstitution peut paraître contradictoire. Pour Jean-Charles Masséra, "la reconstitution est le contraire du remake"; "forme fluide de l'anti-mise en scène", la reconstitution "fonde son principe sur la mise en situation comme critique de la mise en scène" (id., p. 59). II me faut donc préciser, une fois encore, que la répétition dans la différence d'un texte filmique pré-existant s'écarte radicalement de la pratique communément appelée remake. La définition de la re-constitution que nous propose Masséra me paraît rendre compte de la logique à l'œuvre dans Remake: "Dans la forme de la mise en situation, la représentation des faits se présente comme la transmission d'une expérience conscientisée: en tant qu'elle domine tout le principe de la dépossession - sa décontextualisation de distanciation. Pourtant, Pierre Huyghe me semble plutôt activer un processus d'automatisation de la perception du film pour mieux le désamorcer: en dénaturalisant Rear Window, Remake met à nu un mécanisme de réception dans l'inattention du film; pour comprendre le sens de ce qui lui est présenté, le spectateur doit convoquer un souvenir du film étalon comme référent de l'image vidéographique. En tout cas, l'accent porte sur les modalités de réception du film: le spectateur est amené à s'observer, à questionner sa propre pratique de visionnement. Remake constitue un acte réflexif: qu'est-ce qui attire le regard dans un film? Qu'est-ce que percevoir une trame narrative linéarisée ou, en tout cas, des signifiants filmiques? Un "quatrième regard »18, pour reprendre la terminologie de Paul Willemen, est dès lors mobilisé: l'activité spectatorielle est elle-même mise en question, l'œil impassible de la caméra restituant à un public invisible les faux pas d'acteurs réduits au statut d'objets d'étude pour un entomologiste à venir.

Aussi la procédure de sélection de Rear Window est-elle surdéterminée. Une première invisibilité historique a frappé de son sceau le film, l'exploitation des copies (comme pour les autres films réalisés avec James Stewart) ayant été bloquée pour des raisons de droit. Par la suite se greffe une deuxième invisibilité, tout au moins pour une catégorie de spectateurs conscients des différentes strates de discours (critiques, théoriques, cinéphiliques, etc.) qui recouvrent le film. Enfin, Rear Window, dans son fonctionnement interne, ne laisse pas vraiment prise aux mécanismes d'identification du spectateur, l'enchaînement sensorimoteur de l'action étant sans cesse différé. Je m'explique sur ce dernier point: l'autoréférentialité du film est étonnamment grossière pour qui est coutumier des trames psychologiques "ourdies" par Hitchcock et ses scénaristes; l'unité de lieu, d'action et (presque) de temps renvoie à un mode d'exposition pré-cinématographique; et enfin, le mécanisme du champ/contre-champ, qui le plus souvent opère localement, est ici dilaté sur l'ensemble du film. Le caractère déceptif de cette fiction hitchcockienne apparaît avec le plus d'évidence au niveau spectatoriel: le public est en droit de se demander quand on va enfin se décider à passer à l'action.

Dès lors, Pierre Huyghe nous propose un curieux pacte de lecture: en instituant une relation de co-dépendance entre Rear Window et Remake, il court-circuite le texte source par son redoublement dans la différence. Pierre Huyghe opte pour le mode du dialogisme, mais vise un effet de dislocation des voix: un vide généralisé affleure, de part en part gagné par un humour décalé. Les situations paraissent déplacées, les comportements aberrants et les dialogues improbables. Bref, l'abolition 
de l'ordre du vraisemblable s'oppose à la constitution de tout suspense. Pierre Huyghe condamne définitivement, si je puis dire, Rear Window à l'invisibilité. Il ne s'agit pas là d'une opération de déconstruction, mais d'un effacement par recouvrement textuel ou, un peu mieux, d'un arrêt de mort. Il fallait donc que le choix porte sur un objet qui appartient sans équivoque à la culture de masse, à une mémoire (télé)visuelle communément partagée. Se regardant en chiens de faïence, ces objets filmiques coextensifs mais hétéronomes, en attente d'une réactualisation improbable et, somme toute, indésirable, se neutralisent l'un l'autre.

\section{De Remake à une esthétique de la reconstitution}

Il faut, à ce point de l'analyse, marquer le caractère inaugural de Remake dans la fondation d'une "esthétique de la reconstitution»19 qui caractérisera de plus en plus le travail de Pierre Huyghe. Ici, c'est un film policier qui fait l'objet de procédures de démontage. Par la suite, Pierre Huyghe étendra ses investigations à différentes représentations médiatiques et phénomènes de spectacularisation, tout en manifestant une prédilection pour le cinéma sous toutes ses formes (culture de masse, "art et essai», film expérimental).

Lorsqu'il intervient sur des films réputés artistiques, Pierre Huyghe opère des incisions ou des ajouts dans l'original 20. Dans une perspective diamétralement opposée, il se prête à une critique radicale des icônes de la culture de masse et des stratégies de production caractérisant l'industrie du film. Ainsi, avec Blanche neige, Lucie (1997, 4'), il nous propose une «expérience sociologique» $\mathbf{2 1}$ en prenant comme sujet de film le procès intenté à la société Disney par l'actrice qui double la voix de Blanche Neige en français. Filmée sur un plateau de cinéma, Lucie Dolène, d'abord muette, entonne le refrain d'Un jour mon prince viendra qui a rendu sa voix célèbre; des sous-titres, rapportant une conversation avec l'artiste, restituent son histoire. L'issue du jugement authentifie un
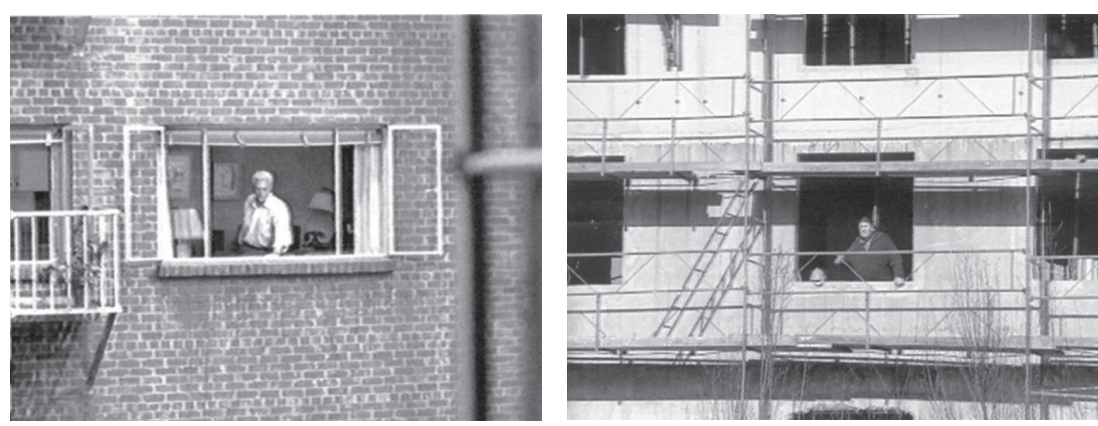

comme sa spectularisation -, et en tant qu'elle ne se sépare plus des commentaires de l'action" (ibid.). La différenciation à opérer dépend de la nature du pré-texte à re-constituer et des effets visés: un film de fiction à dé-naturaliser dans le premier cas, un événement réel à démédiatiser dans le second.

20 Rappelons les principales étapes de ces interventions. Pierre Huyghe, avec Les incivils $\left(1995,40^{\prime}\right)$, revient sur les lieux de tournage de Uccellacci e Uccellini (Des oiseaux petits et grands, Pier Paolo Pasolini, 1966, 88') pour prendre la mesure de la situation socio-économique de I'Italie contemporaine. Avec l'acteur fétiche de Pasolini, Ninetto Davoli, il rejoue certaines scènes, essentiellement les moments de transition; parallèlement, il recueille les témoignages de personnes âgées sur les lieux du tournage; et enfin, ses images captent avec insistance non plus des corps, mais des architectures en chantier et désertées, symboles ossifiés d'une communauté introuvable. Ou encore, dans L'Ellipse (1998, 13'), un système multi-écrans, Pierre Huyghe comble l'ellipse du film d'origine (Der amerikanische Freund, Wim Wenders, 1977, 123'): il invite, en 1998, Bruno Ganz à faire retour sur des lieux laissés inexplorés par la fiction de Wenders (ceux-ci ont "disparu dans la collure"). Enfin, dans Sleeptalking (with Sleep by Andy Warhol and the Voice of John Giorno) $(1998,360$ ' pour la première version ou $3^{\prime}$ en boucle pour la seconde version, avec une bande-son de 60' dans les deux cas), Pierre Huyghe s'empare d'un film du pape de l'underground américain (qui inversait la tradition de la représentation du rêve) pour lui adjoindre une bande-son qui donne à entendre le pionner des spoken words qui sommeille à l'écran. S'il s'agit bien là d'une reprise, les commentaires de John Giorno sur la réalisation de Sleep introduisent une dimension autoréférentielle et réflexive qui modifie le sens du film (ou son absence de sens en lui attribuant de multiples intentions et en actualisant une narration).

21 Je fais évidemment référence au procès intenté par Bertold Brecht contre la Nero Film (incidemment Georg W. Pabst) pour non-respect des droits d'auteur dans l'adaptation de l'Opéra de quat'sous (livret de Bert Brecht, musique de Kurt Weill - qui jette l'éponge après le jugement en première instance). La démonstration de Brecht était la suivante: dans l'industrie du cinéma, la notion d'auteur n'a pas lieu d'être; la Justice, ce dont il ne doutait pas un instant, a confirmé son jugement. 
22 Ajoutons que la figure d'Ann Lee peut être considérée comme le prolongement d'un premier personnage imaginaire conçu par Pierre Huyghe et Philippe Parreno à travers les pages d'un magazine, Anna Sanders. L'histoire d'un sentiment (1996). Le personnage Anna Sanders sera par ailleurs élu comme titre d'appellation d'une société de production de films fondée par Pierre Huyghe, Philippe Parréno et Charles de Meaux.

23 F. Truffaut: "Le MacGuffin, c'est le prétexte, c'est ça?"

A. Hitchcock: "C'est un biais, un truc, une combine, on appelle ça un "gimmick". [...] MacGuffin est donc le nom que l'on donne à ce genre d'actions: voler... les papiers -, voler... les documents -, voler... un secret. Cela n'a pas d'importance en réalité et les logiciens ont tort de chercher la vérité dans le MacGuffin. "

In Hitchcock/Truffaut, François Truffaut (éd.), Editions Ramsay, Paris, 1983, p. 111.

24 Id., p. 178-179. Hitchcock, comme le fait remarquer François Albera, parle dans la bande originale des entretiens d'une expérience menée et décrite par Poudovkine. Mais dans l'édition publiée, les noms de Koulechov et de Mosjoukine ont été introduits! Sur ce point et, plus généralement, sur le mythe de "l'effetKoulechov", voir François Albera, "Koulechov, en effet ", Brûler les planches, crever l'écran. La présence de l'acteur, Gérard-Denis Farcy, René Prédal (éd.), L'entretemps, St Jean-de-Védas, 2001, p. 97-113. acte de violation des droits d'auteur: le spectateur est induit à identifier le doublage d'un personnage d'animation à un processus de réification (Lucie Dolène s'est fait littéralement souffler sa voix). Les interférences entre le monde de la représentation et la sphère privée de l'individu sont aussi au centre de l'installation The Third Memory (2000), une reconstitution avec John Wojtowicz du braquage d'une banque et de la prise d'otages subséquente, fait divers diffusé en direct à la télévision en 1972 et amplifié par la couverture de la presse, qui devient le point de départ d'un film de Sidney Lumet avec Al Pacino (Dog Day Afternoon / Un après-midi de chien, 1975, 129'). Pierre Huyghe interroge les décalages entre la perception subjective de John Wojtowicz (qui avait agi pour couvrir les frais d'opération nécessaires à la transformation de son compagnon en femme), sa médiatisation à travers la presse et la télévision (qui va jusqu'à réunir dans une émission en duplex le braqueur, en prison, et son amant transformé en femme, sur le plateau télévisé), et enfin son accession au titre de cliché à travers un film de fiction. En réactualisant l'événement dans un décor inspiré du film Dog Day Afternoon près de trente ans après les faits, Pierre Huyghe donne la possibilité à John Wojtowicz de forger à neuf une représentation consciente et subjective de sa propre personne. L'acquisition par Pierre Huyghe et Philippe Parreno en 1999 des droits d'un personnage de manga auprès d'une société japonaise d'animation autorise des détournements plus parodiques encore: No Ghost Just A Shell est un projet collectif de production de films avec Ann Lee, personnage fruste et sans histoire, tout en surface, qu'il s'agit de mettre en situation (Pierre Huyghe et Philippe Parreno ont réalisé les deux premiers épisodes qui ont une fonction de prologue) 22.

Toutes ces œuvres mettent en jeu un double mécanisme: le passage d'une personne privée dans une fiction ou dans un phénomène médiatique d'une part, et un geste d'intervertissement ou de renversement des effets de cette publicité (au sens d'appartenance au public) d'autre part. Car il s'agit de se réapproprier une voix, une mémoire ou une individualité, selon les cas. Remake (qui a été réalisé avant ces différentes pièces, soulignons-le) repose sur une opération symétrique mais inversée. Les interprètes s'approprient des personnages filmiques pour les vider de leur substance - les effets induits, par ailleurs, ne sont pas sans liens avec un certain théâtre de l'absurde (notamment avec son attaque frontale contre la subjectivité et la psychologie). Pierre Huyghe met à nu les mécanismes de Rear Window et la vacuité du «MacGuffin»23 - c'était déjà, rappelons-le, l'avis de Hitchcock sur la question. Si Hitchcock peut renvoyer à la conception du montage défendue par Poudovkine $\mathbf{2 4}$ 
et élever la technique du champ/contre-champ au rang de principe de structuration et de déchiffrement du film, Pierre Huyghe dénaturalise les effets de ces codes et démontre que le "prétexte» est une convention éculée. Plus précisément, ce qui intéresse Hitchcock dans cette figure classique de montage, c'est la possibilité de diriger les émotions du spectateur: l'interdépendance établie entre les plans permet de susciter un suspense, qu'il oppose à l'effet de surprise. Ce qui intéresse Pierre Huyghe, dans le retournage de champs/contre-champs avec des moyens techniques frustes et légers, c'est la possibilité d'exhiber et de faire gripper la machinerie filmique: le respect excessif de la symétrie axiale le plus souvent, l'accentuation des angles de prises de vues parfois, et la démultiplication d'une figure féminine par la saute du raccord au moins une fois, ont pour effet d'isoler chaque plan qui raccorde mal avec le précédent et le suivant. La malformation des séquences rompt le charme de l'identification et impose un mode d'observation qui répond à une exhibition des ficelles et des trucs du métier - ce dont Pierre Huyghe a conscience lorsqu'il déclare: «Le film est dépouillé et apparaît donc par moments pornographique» 25. Par "pornographique», il faut à mon sens comprendre: tout montrer en bloquant l'absorption diégétique.

\section{Signes de l'effraction}

Pour revenir plus précisément et localement à Remake, il faut relever que Pierre Huyghe multiplie les variations tant au niveau profilmique que filmique. En premier lieu (celui du profilmique), il met en jeu des mécanismes d'inversion dans l'apparence des personnages: la femme qui interprète Lisa, par exemple, est brune et porte d'autres vêtements que dans l'original. Le décor est stylisé et épuré : un miroir disproportionné (très discret dans l'original) constitue l'élément prégnant de la chambre de Jefferies; et, détail qui a son importance, des photographies de l'immeuble en chantier remplacent les clichés de reportage. Les objets, à
25 Propos rapportés par Catherine Millet, "Les films pornographiques de Pierre Huyghe ", in Art Press, $n^{\circ} 227$, septembre 1997, p. 26-27.
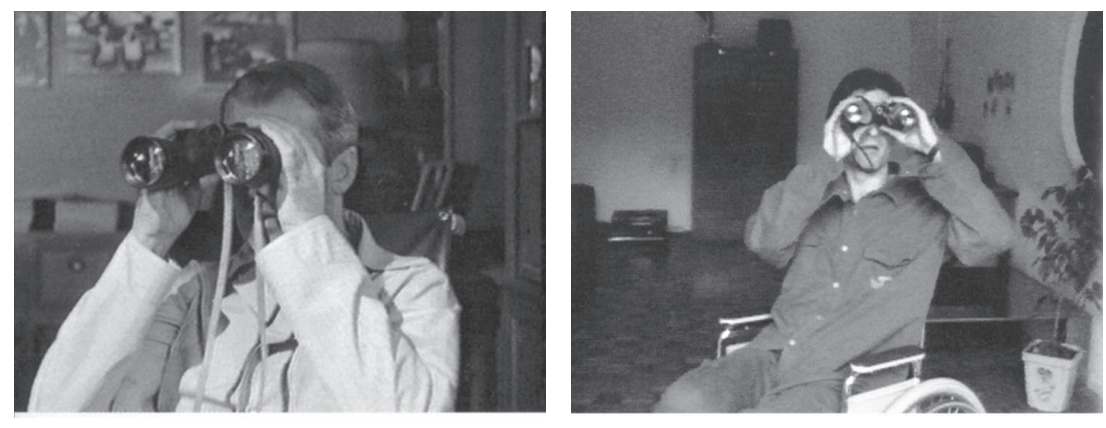
26 Sans prétendre échapper à un mouvement de surinterprétation, je proposerais volontiers l'hypothèse suivante pour expliquer cette substitution: l'échange chien/chat participe d'un mouvement de sexualisation du film. Truffaut et Hitchcock (op. cit.) s'entendaient pour interpréter la mort du chien comme la perte d'un enfant (auquel le couple en question ne pouvait donner naissance en chair et en os). Malgré l'escamotage dans le retournage des signes exhibant le désir de mariage de Lisa, la corporéité de cette dernière est par contre accentuée (elle peut parfois porter des vêtements criants). II me semble possible, dans ces conditions, de rattacher la perte du chat (qui provoque le cri d'effroi de la voisine) à une variation salace sur un proverbe: La nuit, toutes les chattes sont grises... l'inverse de la parabole de Borges, sont modernisés. Ainsi en va-t-il avec le téléphone de Jefferies ou, plus spectaculairement et parodiquement, avec le repas de chez Maxim's (à l'heure de la société de consommation : des produits livrés par Pizza Hut en lieu et place d'un plat fin, du cocacola au lieu d'un cru bourgeois ou encore une cannette en aluminium à la place d'un verre à pied). Les acteurs sont pris au piège: Pierre Huyghe, en bon désorganisateur de la représentation, met en place les conditions d'impossibilité d'une reprise littérale, en provoquant le bégaiement et les hésitations des acteurs qui passent certains éléments du dialogue sous silence ou intervertissent les propos. Par ailleurs, l'acteur qui interprète Jefferies accentue l'état de délabrement de son personnage: sensible au passage du temps, il se présente souvent à l'écran avec une barbe naissante (relevons que lors de sa première apparition éveillé à l'écran, il se rase et que, lors de la querelle avec sa prétendante, il parle de barbe de «trois semaines» au lieu de «trois jours» pour se montrer désagréable). Enfin, Huyghe se prête encore à un jeu de substitution d'éléments du décor et de l'intrigue: un fauteuil remplace le lit où Grace Kelly prenait place et une télévision s'immisce dans les premiers plans, tout comme par la suite résonne la bande-son de Rear Window, off et en version française ; un chat, et non un chien, attire l'attention sur ce qui s'avère constituer les indices du crime. Cette dernière substitution est thématisée dans les dialogues. L'homme qui interprète Jefferies, en comparant une diapositive de broussailles en friche (au lieu de celle d'une plate-bande de fleurs, chez Hitchcock) avec la portion correspondante de la cour, échange les propos suivants :

Jefferies: "Et bien, si je ne me trompe pas, il y a là une preuve du meurtre."

Stella: «De Madame Thorwald?»

Jefferies: "Non, du chien!»

Stella: «Oh! Du chien ou du chat? Du chat!»

Jefferies, Stella et Lisa, en chœur: «Ah! Ah! Du chat! Du chat!»26

En accusant l'erreur de propos et l'incongruité de la substitution, les acteurs indexent le caractère burlesque ou en tout cas potache de cette répétition générale "sans filets».

En deuxième lieu (celui du filmique), les coupes entre les plans se multiplient et les changements d'axe de la prise de vues s'accentuent. Les zooms sur les différentes fenêtres des appartements ne sont pas assez appuyés pour faire voir les bijoux de la victime (des indices pourtant cruciaux) ou encore l'alliance passée au doigt de Lisa (un désir de mariage enfin métonymiquement actualisé). La bande-son, en prise directe, est difficilement audible et ne cherche pas à reconstituer la version 
originale: les dialogues ont tendance à s'effilocher; certains bruits sont omis (le sifflement d'un train lorsque les deux femmes creusent dans le jardin, par exemple) et d'autres sont introduits (un brouhaha de cour de récréation ou une ambiance sonore techno, entre autres); et la musique, interprétée par Björk, semble thématiser ces jeux d'inversion (elle est donnée, extradiégétiquement, dans son intégralité dès le premier plan et ne réapparaît que pendant le générique - alors qu'elle se construit progressivement chez Hitchcock).

Entre-deux, c'est-à-dire à l'intersection du filmique et du profilmique, on assiste à une série de déplacements, principalement en ce qui concerne l'espace. La danseuse (nettement moins déshabillée que dans l'original) fait ses exercices et reçoit ses prétendants dans la cour (et non plus dans un appartement à l'étage); la célibataire éplorée et portée à l'alcoolisme, grimée en clocharde, vit dans la cour (et non plus au rez-de-chaussée de l'immeuble). Et enfin, certains points de vue sont relégués hors-champ: la rue, où pourtant plusieurs personnages censément se rendent, devient un angle mort, provoquant plusieurs ellipses et raccourcis. L'immeuble en chantier, naturalisant la représentation du criminel sous les traits d'un ouvrier, acquiert une double fonction: il devient le signe métafilmique d'un work in progress, et il permet d'ancrer l'espace filmique en un site spécifique. Le jeu sur le cadrage, qui est redoublé par le cadre des fenêtres (il n'y a plus de stores dans l'appartement de Jefferies - qui favorisaient chez Hitchcock un autre jeu avec Lisa en déshabillé, trop rapidement interrompu par un cri de femme), permet de quadriller et de marquer la spécificité de cet espace architectural. Evidemment, le choix d'un lieu en construction résonne avec le travail de Pierre Huyghe et François Roche Chantier permanent (1993) qui documente des habitations aux formes inachevées et provisoires (non soumises aux taxes de construction et construites sans autorisation pendant le temps libre des habitants).
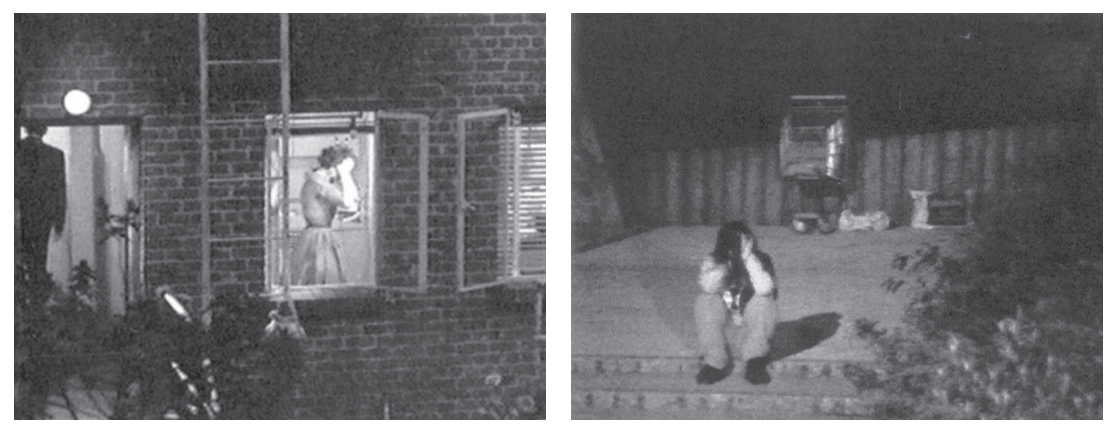
27 Propos rapportés par David Rubbins, "Science-fiction chaude", Pierre Huyghe. Le Château de Turing, Les Presses du réel, 2003 (s. p.).

$28 \mathrm{La}$ structure du palimpseste implique la primauté et la visibilité du texte second. Remake inverse en quelque sorte ce dispositif. Deux œuvres qui participent d'un effet d'illisibilité généralisé me paraissent anticiper la démarche de Pierre Huyghe. Je pense d'une part à Robert Rauschenberg qui, dans un geste iconoclaste, a partiellement effacé une toile de Willem de Kooning dont il ne laisse subsister que quelques rares traces (Erased de Kooning Painting, 1953 - l'opération lui a quand même pris quatre semaines!). Et je pense d'autre part à Marcel Broodthaers qui a reproduit en creux la disposition graphique sur la page des vers du Coup de dés (Stéphane Mallarmé) tout en élidant ses signes typographiques (Un coup de dés jamais n'abolira le hasard, 1969, 25 pages).

Remerciements: Galerie Marian Goodman, New York / Paris
La séquence de clôture affirme sa divergence d'avec l'original, tout en accentuant la spécificité du site exploré : l'immeuble paraît déserté, rejetant hors-champ les différentes résolutions imaginaires des histoires des locataires. Pierre Huyghe prend congé des spectateurs en laissant les lieux du crime vacants et les décors du film inachevés. Le coupable est un ouvrier du bâtiment: son seul crime consiste à s'être prêté à des travaux d'échafaudage provisoires et sans permis. La boucle est ainsi bouclée: Pierre Huyghe, à l'image de Lisa, s'introduit par effraction dans un espace non autorisé et finit par bouter l'infortuné hors des lieux. Et ce n'est pas un hasard si l'intégralité du métrage de Remake s'apparente à un chantier illicite, un lieu qui se construit anarchiquement.

Mais laissons le mot de la fin à Pierre Huyghe qui nous convie fort consciemment à une réjouissante mascarade où les rôles et les fonctions filmiques s'intervertissent:

«Le Capitalisme, peut-il ainsi avancer, éprouve une véritable fascination envers les faiseurs d'histoires (événement) - des histoires que nous écoutons et regardons, puis racontons et jouons, des histoires qui sont signées d'un nom et célébrées. Jouer avec des rôles créés par le monopoly narratif est sain: le narrateur (celui qui raconte) et le narré (celui qui écoute l'histoire) doivent s'inverser ou s'annuler. »27 En réduisant Rear Window à ses seuls soubassements narratifs (sans glamour hollywoodien ou nostalgie pour ce faste), Pierre Huyghe remet en cause la non communication entre les places assignées par l'industrie du divertissement au réalisateur, à l'acteur et au spectateur. Le côté arte povera de Remake provoque un mouvement d'indifférenciation entre la sphère privée de l'individu et la sphère publique de la profession. On est donc aux antipodes du geste de Gus van Sant qui re-filme en couleurs et non sans moyens techniques Psycho (1999) - qui reproduit (avec glamour et nostalgie) une œuvre déjà intégrée au règne du multiple.

En fin de compte, il n'est plus possible de déterminer quel film constitue le prétexte de l'autre. Le paradoxe qui frappait le film de Hitchcock, celui de capitaliser un record d'entrées de spectateurs tout en étant condamné à l'invisibilité pendant plus de vingt ans, est remis en jeu par Pierre Huyghe qui attribue par la même occasion une nouvelle visibilité à Rear Window. La structure du palimpseste est dès lors inversée: c'est Hitchcock qui recouvre le texte huyghien, qui pourtant s'offrait comme supplément de Rear Window ${ }^{\mathbf{2 8}}$. A travers ce miroir aux alouettes, c'est la vraisemblance qui s'abîme à jamais. 


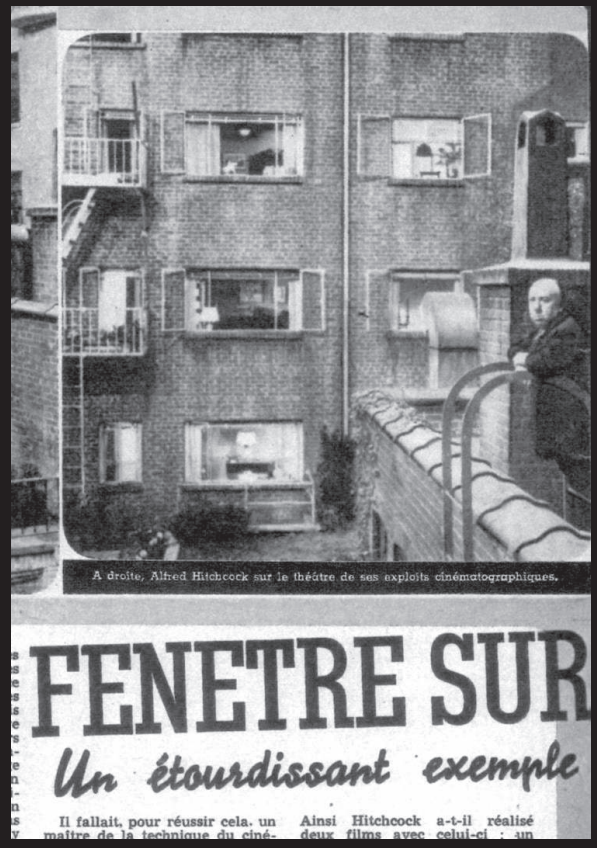

À L'ÉPOQUE DE LA PREMIÈRE SORTIE FRANÇAISE DE FENÊTRE SUR COUR (DÉBUT 1955), UN MAGAZINE POPULAIRE DE CINÉMA LUI CONSACRE UNE PLEINE PAGE EN SOULIGNANT DEUX ASPECTS: LA "VIRTUOSITÉ " CINÉMATOGRAPHIQUE DU CINÉASTE (PRÉSENT À L'IMAGE) ET LA VALEUR D'ATTRACTION DES "VIES INTIMES" DÉVOILÉES PAR LE TÉLÉOBJeCtIF. 


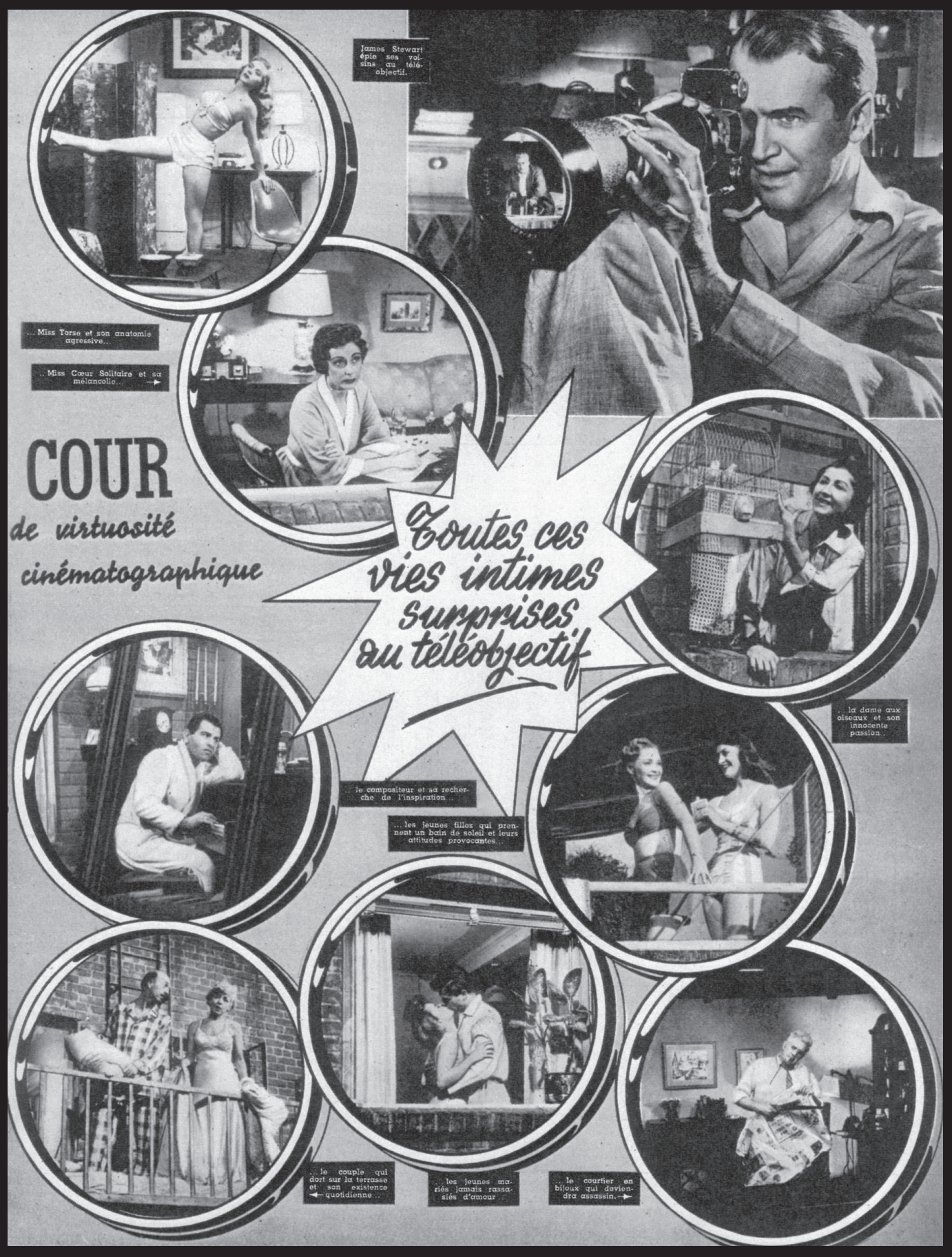

